J Sci.Univ.Kelaniya 4 (2008): 68-72

\title{
PHYSICAL INTERPRETATION OF ANOMALOUS ABSORPTION OF PARTIAL WAVES BY NUCLEAR OPTICAL POTENTIAL
}

\author{
R. A. D. PIYADASA, J. MUNASINGHE AND N. G. A. KARUNATILEKE \\ Department of Mathematics, University of Kelaniya, Kelaniya, Sri Lanka. \\ E-mail : piyadasa54@yahoo.com
}

\begin{abstract}
Anomalous absorption of light ion partial waves by the nuclear optical potential is an interesting phenomenon showing striking systematic in various parameter planes. However, the theoretical description of this phenomenon is extremely difficult. In this contribution, we address the problem of its physical interpretation. It is shown that the zero of the S-matrix element associated with the anomalous absorption of a partial wave is due to the destructive interference of the reflected waves, from the innermost turning point and the outermost turning point, in the asymptotic region when WKB approximation is feasible.
\end{abstract}

Key words: anomalous absorption, Partial waves, S-matrix element.

\section{INTRODUCTION}

Anomalous absorption of partial waves by the nuclear optical potential is an interesting phenomenon since the zero corresponding to anomalous absorption of partial wave is due to positive energy. This phenomenon was first discovered in case of elastic scattering of neutrons on composite nuclei (Kawai \& Iseri, 1985; Iseri \& Kawai, 1986) and it has been found (Piyadasa, 1986) that the phenomenon is universal for light ion elastic scattering on composite nuclei. It has been found that, in case of light ion elastic scattering on composite nuclei, the S-matrix element becomes so small that it does contribute to the differential cross-section as S-matrix element were zero. 
This occurs for a special combination of energy (E), angular momentum ( $l$ ), total angular momentum $(j)$ and the composite target nuclei (A). Complete theoretical description of this phenomenon is extremely difficult. However, we have found that the physical interpretation of this phenomenon can be done using WKB approximation corresponding to three turning points (Brink \& Takigawa, 1976). We have found that the zero of the S-matrix element corresponding to a partial wave of angular momentum $(l)$ is due to the destructive interference of the reflected waves due to the innermost turning point and the outermost turning point in the asymptotic region. This interpretation is justified theoretically with the supporting numerical results in the following sections.

\section{MATERIALS AND METHODS}

We use the method of semi-classical approximation, well known as WKB approximation, to justify our claim that the anomalous absorption of a partial wave is due to the destructive interference of reflected waves in the asymptotic region. A formula for semi-classical elastic $\boldsymbol{S}$-matrix element has been derived by Brink \& Takigawa (1976) for a potential having three turning points with a barrier. If $S_{l j}$ denotes the $S$-matrix element corresponding to angular momentum $l$ and total angular momentum $l, S_{l j}$ is given by

$$
S_{l j}=\exp \left(2 i \delta_{1}\right) \frac{1+\bar{N}(i \varepsilon) \exp \left(2 i S_{32}\right)}{N(i \varepsilon)+\exp \left(2 i S_{32}\right)}
$$

where $N(z)$ is defined by

$$
N(Z)=\frac{\sqrt{2 \pi}}{\Gamma\left(\frac{1}{2}+Z\right)} \exp \left(Z \ln \left(\frac{Z}{e}\right)\right) \text { and } s=\frac{-t}{\pi} S_{21} \text { in the usual notation. }
$$

In particular $S_{21}, S_{92}$ are action integrals corresponding to the turning points $\left(r_{1}, r_{2}\right)$ due to the potential barrier and turning points $\left(r_{2}, r_{3}\right)$, where $r_{3}$ is the inner most turning point and $r_{2}$ is the turning point between $r_{1}$ and $r_{3}$.

If $\kappa=\sqrt{\frac{2 \mu E}{\hbar^{2}}}$ is the incident wave number associated with the projectile light ion, it can be shown that (Piyadasa \& Mallawa Arachchi, 2005)

$$
1+\frac{1+\exp (2 \pi \varepsilon)}{N(i \varepsilon)} \exp \left(2 i S_{31}\right)=0
$$


if $s_{l j}(k)=0$. From (2), one obtain, at once

$$
S_{31}=(2 n+1) \frac{\pi}{2}+\frac{1}{2 i} \ln \left(\frac{N(i \varepsilon)}{1+\exp (2 \pi \varepsilon)}\right)
$$

which is a necessary and sufficient condition for the semi-classical $S$-matrix element to be zero. Obviously, $S_{l j}(k)=0$ means the absence of an outgoing wave since the asymptotic wave boundary condition for the corresponding partial wave $U_{i f}(k, r)$ is given by

$U_{i j}(k, r) \sim U_{i}^{(-)}(k, r)-S_{i f}(k) U_{i}^{(+)}\left(k_{r} r\right)$

where $U_{l}^{(-)}$and $U_{l}^{(+)}$stand for the incoming and outgoing Coulomb wave functions respectively. In case of a bound state due to a real potential with two turning points $\left(x_{1}, r_{2}\right)$, it is well known that

$\int_{r_{2}}^{n_{2}} p(r) d r=(2 n+1) \frac{\pi}{2}$

where $p(x)$ is the momentum and $n$ is the number of nodes of the wave function within $\left(r_{1}, r_{2}\right)$. We claimed (Piyadasa \& Mallawa Arachchi, 2005) that the zero of semi-classical $S$-matrix element satisfies the condition that

$\frac{N(i \varepsilon)}{1+\exp (2 \pi \varepsilon)} \approx 0$

and then

$$
S_{01}=(2 n+1) \frac{\pi}{2}
$$

This can be understood by the expression

$$
S_{i j}=\frac{e^{2 i \delta 2}}{N(i \varepsilon)}+\frac{e^{2 i \delta 3}}{N(i \varepsilon)\left(N(i \varepsilon)+e^{2 i S_{3}}\right)}
$$

if one assumes that $N(t a) \sim \mathbb{1}$ and $\boldsymbol{s}^{2 t \delta_{s} \sim 0}$, which are reasonable assumptions if the three-point WKB approximation is feasible. Then the $S$-matrix becomes

$$
\begin{aligned}
& S_{i j}(\kappa)=\frac{e^{2 i \delta_{1}}}{N(i \varepsilon)}+\frac{e^{2 i \delta_{3}}}{N(i \varepsilon)} \\
& S_{i j}(\kappa)=n_{\beta}+n_{l}
\end{aligned}
$$

where $n_{g}$ and $n_{I}$ stand for the amplitude of the reflected wave due to the external turning point and the amplitude of the reflected wave due to the inner most turning point respectively. Then it is clear that $s_{t z}(k)=0$ is due to the fact that the destructive interference of reflected waves in the asymptotic region. In particular, if $S_{i j}(k)=0$, it follow from (8) that 
$S_{\mathrm{a} 1}=(2 n+1) \frac{\pi}{2}$

which is the well known Bohr-Sommerfeld quantum rule.

\section{RESULTS}

We have found that the partial wave $E=10$ is almost completely absorbed in case of ${ }^{4} \mathrm{He}$ elastic scattering on ${ }^{40} \mathrm{Ca}$ at the centre of mass energy of $25.72 \mathrm{MeV}$. Turning points corresponding to $l=10$ are

$$
\begin{aligned}
& r_{1}=6.71594-t 1.2226, \quad r_{2}=6.68966+t 1.30959, \quad r_{1}=1.81386-t 0.078712 \\
& S_{81}=11 \frac{\pi}{2}+t 0.04356, \quad K(t a)=1.0026+t(.054710 \\
& \left|e^{2 i S_{32}}\right|=e^{-5.37}
\end{aligned}
$$

If should be noted that the calculation is carried out using Mathematica and we have approximated the coulomb potential by an analytic function of $r$ which agree with the exact coulomb potential only ninety five percent. Even then the above numerical results clearly manifest the fairness of our assumptions. The following figure, corresponding to ${ }^{4} \mathrm{He}+{ }^{40} \mathrm{Ca}$ elastic scattering at the centre of mass energy of $25.72 \mathrm{MeV}$.

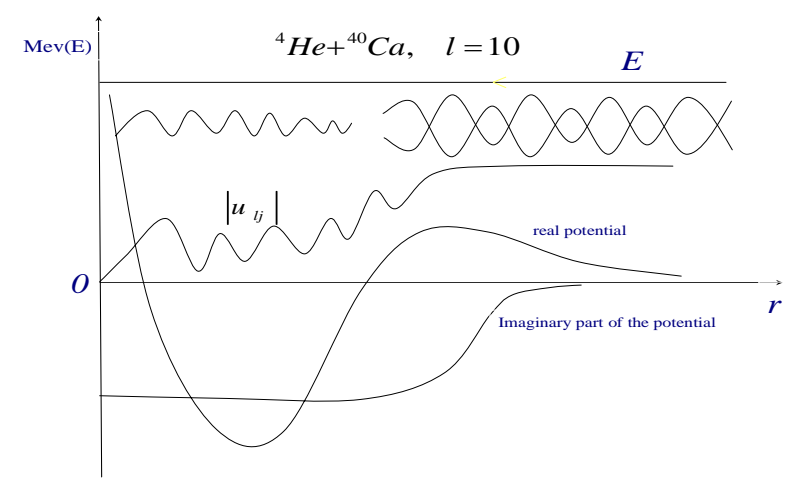

In the figure $\left|u_{l j}\right|$ denotes the modulus of the partial wave function absorbed by the nuclear optical potential, which does not oscillate in the asymptotic region since the outgoing part of the wave is absent. The reflected waves at the innermost and at the outermost turning points are in the destructive interference in the asymptotic region 
and this is shown schematically in the figure. $E$ denotes the centre of mass energy of the ${ }^{4} \mathrm{He}$ projectile.

\section{DISCUSSION}

Theoretical description of all features of the anomalous absorption of partial waves by the nuclear optical potential seems extremely difficult. However, its physical meaning is now clear, at the same time that the number of nodes, of an absorbed partial wave, inside the potential has a relation to the systematic in the $\left(R_{c}, A^{1 / 3}\right)$ plane, where $R_{c}$ is the well known closest approach, such that all partial waves of the same node lie on the same straight line in $\left(R_{C}, A^{1 / 3}\right)$ plane. This point also may be understood.

\section{REFERENCES}

Brink, D.M. \& N. Takigawa. 1976. Barrier penetration effects in the semi classical theory of elastic scattering between complex nuclei, Nuclear Physics A279 : 159-188.

Iseri, Y. \& M. Kawai. 1986. Anomalous absorption of portion partial waves by the optical potential, Physical Review C 34 : 38-44.

Kawai, M. \& Y. Iseri. 1985. Anomalous absorption of neutron partial waves by the nuclear optical potential, Physical Review C 31 : 400-408.

Piyadasa, R.A.D. 1986. Master Thesis submitted to the University of Kyushu, Japan.

Piyadasa, R.A.D. \& D.K. Mallewa Arachchi. 2005. Annual Research Symposium, Faculty of Graduate Studies, University of Kelaniya, Sri Lanka (Abstract) 74. 